\title{
Serum testosterone levels and symptom-based depression subtypes in men
}

\author{
Stephanie Rodgers ${ }^{1}$, Martin grosse Holtforth ${ }^{2,3}$, Michael P. Hengartner ${ }^{4}$, Mario Müller ${ }^{1}$, \\ Aleksandra A. Aleksandrowicz ${ }^{1}$, Wulf Rössler ${ }^{1,5,6}$ and Vladeta Ajdacic-Gross ${ }^{1 *}$ \\ ${ }^{1}$ Department of Psychiatry, Psychotherapy and Psychosomatics, Zurich University Hospital of Psychiatry, Zurich, \\ Switzerland, ${ }^{2}$ Department of Psychology, University of Zurich, Zurich, Switzerland, ${ }^{3}$ Department of Psychology, University of \\ Bern, Bern, Switzerland, ${ }^{4}$ Department of Applied Psychology, Zurich University of Applied Sciences, Zurich, \\ Switzerland, ${ }^{5}$ Collegium Helveticum, Swiss Federal Institute of Technology, University of Zurich, Zurich, \\ Switzerland, ${ }^{6}$ Laboratory of Neuroscience (LIM27), Institute of Psychiatry, University of São Paulo, São Paulo, Brazil
}

\section{OPEN ACCESS}

Edited by: Shervin Assari,

University of Michigan, USA

Reviewed by:

Stephane Rothen,

Geneva University Hospitals,

Switzerland

Mohammadreza Mokhtari, Hartford Hospital, USA

*Correspondence:

Vladeta Ajdacic-Gross,

Department of Psychiatry, Psychotherapy and Psychosomatics,

Zurich University Hospital of Psychiatry, PO Box 1930, Zurich $\mathrm{CH}-8021$, Switzerland vajdacic@dgsp.uzh.ch

Specialty section:

This article was submitted to Public Mental Health, a section of the journal

Frontiers in Psychiatry

Received: 11 November 2014 Accepted: 09 April 2015 Published: 04 May 2015

Citation:

Rodgers S, grosse Holtforth $M$ Hengartner MP, Müller M, Aleksandrowicz AA, Rössler W and Ajdacic-Gross V (2015) Serum testosterone levels and symptom-based depression subtypes in men.

Front. Psychiatry 6:61. doi: 10.3389/fpsyt.2015.00061
The main objective of this preliminary study was to further clarify the association between testosterone ( $\mathrm{T}$ ) levels and depression by investigating symptom-based depression subtypes in a sample of 64 men. The data were taken from the ZInEP epidemiology survey. Gonadal hormones of a melancholic $(n=25)$ and an atypical $(n=14)$ depression subtype, derived from latent class analysis, were compared with those of healthy controls $(n=18)$. Serum T was assayed using an enzyme-linked immunosorbent assay procedure. Analysis of variance, analysis of covariance, non-parametrical tests, and generalized linear regression models were performed to examine group differences. The atypical depressive subtype showed significantly lower T levels compared with the melancholic depressives. While accumulative evidence indicates that, beyond psychosocial characteristics, the melancholic and atypical depressive subtypes are also distinguishable by biological correlates, the current study expanded this knowledge to include gonadal hormones. Further longitudinal research is warranted to disclose causality by linking the multiple processes in pathogenesis of depression.

Keywords: testosterone, depression, subtypes, epidemiology, cross-sectional study

\section{Introduction}

Apart from its gonadal functions, testosterone (T) has a significant influence on the human brain through various neurobiological processes (1-3). In men, associations between $\mathrm{T}$ levels and depressive symptoms have been proposed $(3,4)$. Such neuroendocrine dysfunctions may play an important role in the pathogenesis of major depressive disorder (MDD) (5). In particular, low T levels (hypogonadism) have been associated with depression $(4,6-8)$, and there is evidence that $\mathrm{T}$ secretion is impaired during depressed mood $(9,10)$. However, this association has not been observed consistently and some studies did not find any relationship between $\mathrm{T}$ levels and depression $(11,12)$. These inconsistent findings could be explained by the heterogeneity of the construct of MDD (13) or difficulties concerning the measurement of salivary T versus that of serum $\mathrm{T}$ (14). Moreover, many findings stem from studies involving exogenous $\mathrm{T}$ administration, whereas studies looking at the effects of endogenous $\mathrm{T}$ are lacking (15). In sum, there appears to be insufficient evidence to conclude that low $\mathrm{T}$ levels are routinely involved in the pathogenesis of MDD in men (15). 
However, a growing body of evidence suggests that there may be subpopulations of men vulnerable to depression in which hypogonadism is a contributing factor (16). Some studies showed that low $\mathrm{T}$ levels were specifically associated with subthreshold depressive disorders, such as dysthymia or minor depression (15, 17-20). Other studies demonstrated that hypogonadism was related to the specific depressive symptoms of dysphoria, irritability, fatigue, lethargy, decreased libido, and decreased concentration $(6,15)$. This symptom cluster has also been conceptualized as "irritable male syndrome," occurring in adult male mammals following withdrawal of $\mathrm{T}$ (21).

Meanwhile, some investigations have drawn attention to nonlinear associations between total $\mathrm{T}$ levels and depressive symptoms, with increased depression rates at both the lowest and the highest extremes of T levels $(15,22)$. Furthermore, exogenous $\mathrm{T}$ administration has not only been related to depression but also to hypomania and even mania (23-28). Despite these suggestive findings, to date, the associations between dysregulated endogenous T levels and affective symptoms remain fairly unclear (15).

Apart from the findings with regard to the hypothalamicpituitary-gonadal (HPG) axis, previous studies have investigated associations between atypical and melancholic (typical) depression and the hypothalamic-pituitary-adrenal axis (HPA), the inflammatory response system and metabolic abnormalities $(29,30)$. Melancholic depression has been characterized by hypercortisolemia (HPA axis), whereas atypical depression has been associated with the opposite imbalance, namely hypocortisolemia (31). The HPA axis also interacts with gonadal hormones $(32,33)$ and Sigurdsson et al. (34) found a positive correlation between evening cortisol and evening $\mathrm{T}$ levels. Therefore, one might hypothesize that melancholic depression is associated with high $\mathrm{T}$ levels and atypical depression shows the opposite, that is to say low $\mathrm{T}$ levels. Moreover, $\mathrm{T}$ levels are negatively correlated with the body mass index (BMI) (35), and atypical depression is characterized by a higher BMI (36), further supporting the hypothesis. Consequently, the melancholic and atypical depression subtypes might not only be distinguishable by differing activity of the HPA axis but by that of the HPG axis as well.

In view of the inconsistency of the findings summarized above, this preliminary study set out to examine the differences in serum $\mathrm{T}$ levels in symptom-based depression subtypes, compared with healthy controls. The classification of these depression subtypes was based on a previous latent class analysis, derived from the depressive symptom profiles of an epidemiological sample of males (37). The purpose of this paper is to provide an alternative approach to the area of research by analyzing $\mathrm{T}$ levels in empirically derived depression subgroups. Based on previous findings with regard to $\mathrm{T}$ and depression and by drawing analogies to the HPA axis, we expected differing T levels between these depressive subgroups, with higher $\mathrm{T}$ levels for the melancholic depression subgroup and lower T levels for the high BMI associated, atypical depression subgroup.

\section{Materials and Methods}

\section{Study Design and Sampling}

Data from the epidemiology survey of the Zurich Program for Sustainable Development of Mental Health Services (ZInEP; German:

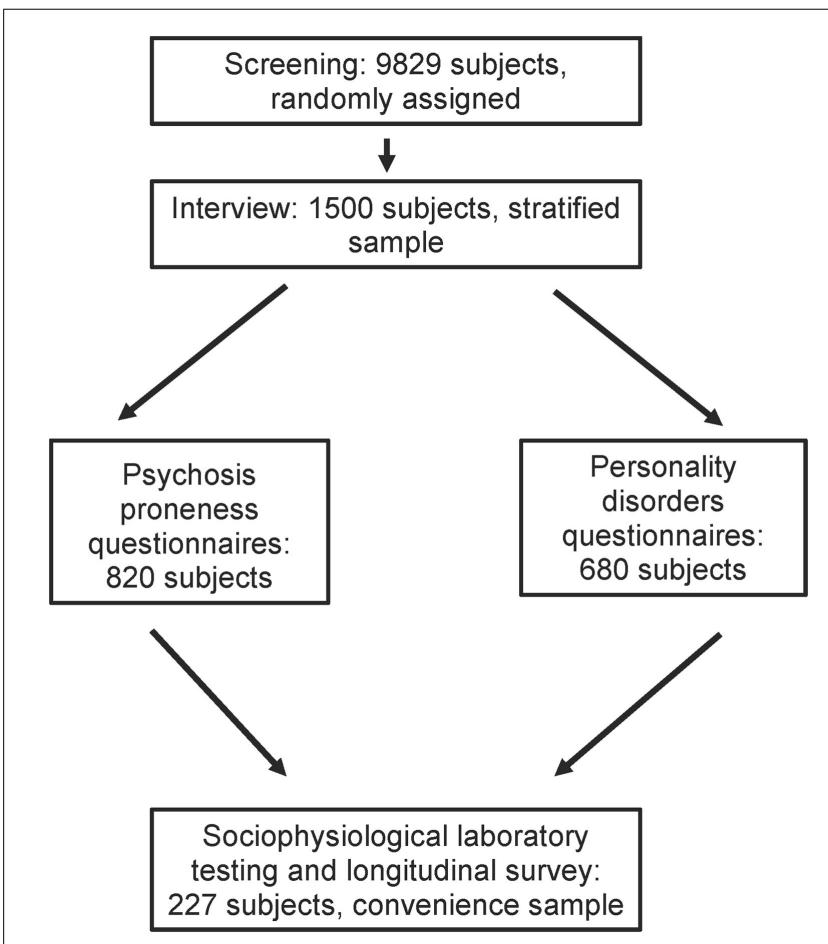

FIGURE 1 | The sampling procedure of the ZInEP epidemiology survey

Zürcher Impulsprogramm zur nachhaltigen Entwicklung der Psychiatrie) were used. The epidemiology survey was conducted in order to collect comprehensive data about mental health in the general population of adults in the canton of Zurich. The survey was methodologically designed as a cross-sectional sequel to the prospective Zurich cohort study (38), e.g., the instruments and the age structure were parallelized. It consisted of three components: (a) a brief telephone screening ( $n=9829)$, (b) a structured face-to-face-interview supplemented by self-report questionnaires $(n=1500)$, and (c) a laboratory day followed by a longitudinal survey $(n=227)$ (see Figure 1). The survey was carried out between August 2010 and September 2012. In the following, only the laboratory day (c) providing the biological data will be elucidated. For more details with regard to (a) and (b), see Ajdacic-Gross et al. (39).

For the laboratory day, a subsample of 227 subjects was selected from the face-to-face sample $(n=1500)$. Sample construction was based on the outcomes of two psychoticism scales [schizophrenia nuclear symptoms scale (SNS) and schizotypal signs scale (STS) (40)], which were included in the screening interview, as follows: highest quintile on both scales; highest quintile on the SNS, any quintile on the STS; highest quintile on the STS, any quintile on the SNS; controls with a low overall global severity index (GSI) (41); and no use of professional help. The participation rate was $53.8 \%$ and depended mainly on the ease of acquiring the subjects for the time-consuming set of tests performed during a day in the sociophysiological laboratory of the Zurich University Hospital of Psychiatry. The participants were interviewed, provided saliva, blood, and urine samples, completed computer based tests, and underwent several tests involving electroencephalographic (EEG) and near infrared spectroscopy (NIRS) measurement. All tests 
were carried out by a biologist and three assistants (psychology students). Physicians from the Zurich University Hospital of Psychiatry were involved in the procedure of blood sampling. The gonadal hormones were exclusively derived from the blood probes. However, no gonadal hormone assays were taken from 37 subjects. The reasons for this were participants refusing blood sample collection or difficulties during blood sampling (e.g., problematic positions of veins). Of the 190 participants (men: $n=89$; women: $n=101$ ) with complete hormone data, 137 subjects (72.1\%) were initially allocated to the stratum of high-scorers, while 53 subjects (27.9\%) were low scorers.

The ZInEP epidemiology survey study was approved by the Ethical Committee of the canton of Zurich (KEK) and is in accordance with the latest version of the Declaration of Helsinki. After being extensively informed about study procedure and aims both verbally and in writing, the participants gave their written consent.

\section{Measurements \\ Depressive Symptom Subtypes}

Depressive symptom subtypes were assessed by a computerassisted version of the Structured Psychopathological Interview and Rating of the Social Consequences of Psychological Disturbances for Epidemiology (SPIKE) used in the Zurich Study (38, 42). This instrument includes the most common psychopathological syndromes/disorders. The validity and reliability of the SPIKE have been established for the assessment of depression and anxiety (38). For the current study, we made use of the three class solution, which had previously been extracted by a datadriven technique (latent class analysis, LCA) from the SPIKE data of 373 men manifesting depressive symptoms during the past 12 months (37): a melancholic depression subtype $(n=219$; $58.7 \%)$, an atypical subtype $(n=64 ; 17.2 \%)$, and a moderate subtype $(n=90 ; 24.1 \%)$. We excluded the moderate subtype because the investigation of the two clear discriminable melancholic and atypical depression subtypes increased statistical power, which was necessary due to the small sample sizes. The melancholic and atypical depression subtypes are in line with previous subtyping approaches and/or the DSM-5 specifiers (36, 43-47). The distinction of these two subtypes on the basis of appetite-/weight related symptoms was particularly striking. Members of the atypical subtype showed high probabilities of increased appetite and weight gain, whereas the melancholic subtype was characterized by loss of appetite and weight. More detailed information about these depressive subtypes and the entire LCA procedures can be found elsewhere (37).

Overall, the T data were available for $n=89$ men. From this group, 39 subjects either matched with the melancholic or the atypical depression subtype of the LCA three class solution (37). A subgroup of 18 controls (defined without use of professional help and low overall GSI-scores) was included. Consequently, the present study was dealing with a sample of 57 subjects.

\section{Psychosocial Characteristics}

In order to characterize the depression subtypes, sociodemographic variables such as age, education, urbanicity, and marital status were drawn from the face-to-face interview. Axis I diagnoses were computed according to DSM-III-R/IV criteria for the time-period of the past 12 months $(48,49)$. Only the diagnosis of neurasthenia was derived from ICD-10 (50). Due to methodological linkage with the Zurich Study (38), the algorithms of the DSM diagnoses were parallelized. As a consequence, some diagnoses were based on an older edition of the DSM. Psychosis syndromes were derived according to the algorithm of Rössler et al. (40); bipolar disorder was derived in analogy to the BRIDGE study (51). Personality disorders were assessed using the German translation of the assessment of DSM-IV personality disorders questionnaire (ADP-IV) $(52,53)$, showing good concordance with the SKID-II semi structured interview (54). The current smoking status and the GSI were derived from the CATI. Finally, the data concerning the consumption of psychiatric medication were taken from the laboratory day.

\section{Biological Measures \\ Hormones}

In order to ensure consistency due to diurnal variation (6) and in accordance with the recommendation of an earlier study (55), the blood sample was drawn in the morning. The exact time frame of the blood sample collection lay between 08:55 a.m. and 10:34 a.m., 01:45-03:51 h after the subject's awakening following an overnight fast. Blood was centrifuged ( $10 \mathrm{~min} ; 3000 \mathrm{rpm}$ ) $30 \mathrm{~min}$ after the sample was taken and stored at $-80^{\circ} \mathrm{C}$ until delivered to the CYTOLAB for biochemical analysis. Total $\mathrm{T}$ was derived from serum and measured by enzyme-linked immunosorbent assay (ELISA) using a kit from IBL International, Hamburg, Germany. Dehydroepiandrosterone-sulfate (DHEA-S), luteinizing hormone (LH), estradiol, and progesterone were also derived from serum. While DHEA-S, estradiol, and progesterone were likewise assayed by ELISA (DHEA-S: IBL International, Hamburg, Germany; estradiol and progesterone: BioCheck, Foster City, USA), LH was measured by Bead-Assay (Millipore, Zug, Switzerland). Sex hormone-binding globulin (SHBG) was derived from ethylene diamine tetraacetic acid (EDTA) plasma and measured by ELISA using a kit from IBL International, Hamburg, Germany.

\section{Blood pressure and BMI}

Blood pressure was measured on the left upper arm in a sitting position at the beginning of the laboratory assessment and $15 \mathrm{~min}$ later with the Boso Medicus Prestige (Bosch + Sohn GmbH \& Co, KG DE). Both systolic and diastolic values were obtained and averaged across the two measurements. BMI was conventionally calculated, based on mass and height $\left(\mathrm{kg} / \mathrm{m}^{2}\right)$.

\section{Statistical Analysis}

All data were analyzed using SPSS version 20 for Macintosh (IBM Corp., Armonk, NY, USA). The criterion for significance was set at $p<0.05$.

First, $\mathrm{Chi}^{2}$-tests and Fisher exact tests (two-tailed) and nonparametric Kruskal-Wallis tests were performed in order to assess differences of psychosocial characteristics between the depressive subgroups atypical depression, melancholic depression, and controls.

Second, unadjusted analyses of variance (ANOVAs) were performed to evaluate the between-group differences on metric dependent variables. Before performing the ANOVAs, Levene's 
tests were computed in order to examine the homogeneity of variances, and Shapiro-Wilk tests were used to account for the assumption of normally distributed residuals. The values of $\mathrm{T}$ were $\log$ transformed (natural logarithm, $\ln$ ) due to the non-normally distributed residuals of the control group. In the ANOVAs, the depressive subgroup served as independent variable and the biological characteristics were treated as the dependent variable. Corresponding effect sizes were reported in terms of partial $\eta^{2}$ [0.01 $=$ small, $0.06=$ medium, $0.14=$ large; (56) $]$. In case of significant main effects, Scheffé and Games-Howell post hoc comparisons were used to explore all possible pairwise subgroup comparisons. Categorical biological characteristics were compared between the subgroups with the Fisher's exact test for categorical variables (two-tailed). In addition, a non-parametric median test was carried out on the $\mathrm{T}$ data. Finally, an analysis of covariance (ANCOVA) considering the influence of the covariates age, smoking, alcohol use, and psychopharmacological medication was computed.

Third, the subgroup differences were further examined by generalized linear regression models (GLMs) using logistic regression. GLMs enable the use of robust estimators in order to minimize the effects of outliers and influential observations (57). T was entered as independent variable and the subgroups served as dependent variable.

\section{Results}

\section{Psychosocial Characteristics}

In Table 1, the psychosocial characteristics of the LCA-based depressive subtypes and the control sample are displayed. No significant differences in demographic correlates were observed between these subsamples. However, groups differed with regard to some comorbid 12-month diagnoses. As expected, both depressive subtypes had higher frequencies of MDD diagnosis than the controls. The severe atypical subtype revealed a diagnosis of generalized anxiety disorder (GAD) and simple phobia significantly more often than the controls. In comparison with the melancholic subtype, agoraphobia occurred significantly more often in the atypical depression subtype. Atypical depression showed significantly more psychosis syndromes and personality disorders than the melancholic depression subtype and the controls. Antidepressant consumption was significantly associated with the atypical depression subtype (versus controls). Finally, in terms of the subjectively rated burden related to health problems, both depressive subtypes showed significantly higher values compared with controls.

\section{Biological Characteristics}

The biological characteristics of the subsamples are summarized in Table 2. The following T means and standard errors (SEs) (in $\mathrm{ng} / \mathrm{ml})$ were observed: atypical subtype: $3.92(0.37)$, melancholic subtype 5.33 (0.28), and controls 4.87 (0.33). Log transformed data were used for computing the ANOVA. The atypical depression subtype differed significantly from the melancholic subtype with regard to lower T levels. The difference with respect to the controls remained on a trend level of significance. The same subgroup differences were found for SHBG. Furthermore, men manifesting atypical depressive symptom configurations were significantly more prone to a higher BMI than the melancholic depression subgroup and controls, respectively. The corresponding effect sizes of T, SHBG, and BMI were large.

In an extension of the analyses, the biological factors were categorized in order to validate the results in terms of medically relevant cut-offs: hypogonadism, which was defined as $<2.3 \mathrm{ng} / \mathrm{mL}$ (58), occurred in $n=2(14.3 \%)$ men belonging to the atypical depression subtype and the overall group differences could only be expressed as trend level associations $\left(\chi^{2}=4.119, d f=2\right.$, $p=0.06$ ). The categorized BMI (normal weight: BMI 18.50-25.00; overweight: BMI 25.01-30.00; obesity: $\geq 30.01)$ showed significant overall differences $\left(\chi^{2}=12.435, d f=4, p<0.05\right)$ with significantly differing frequencies between the atypical depression subtype [normal weight: $n=5$ (38.5\%); overweight: $n=4(30.8 \%)$; obesity: $n=4(30.8 \%)]$ and the melancholic depression subtype [normal weight: $n=21$ (84.0\%); overweight: $n=4$ (16.0\%); obesity: $n=0(0.0 \%)$ ], and controls [normal weight: $n=13(72.2 \%)$; overweight: $n=5(26.8 \%)$; obesity: $n=0(0.0 \%)$, respectively. The categorized blood pressure revealed no single cases of hypotension (defined as $<90 / 60 \mathrm{mmHg}$ ). In contrast, hypertension (defined as $>140 / 90 \mathrm{mmHg}$ ) occurred most frequently in the melancholic depression subtype $(n=8 ; 72.7 \%)$. However, the overall differences failed to achieve a customary level of statistical significance $\left(\chi^{2}=4.539, d f=2, p=0.107\right)$ (data not tabulated).

The non-parametric median test provided significant overall subgroup differences regarding the T levels $\left(\chi^{2}=6.685, d f=2\right.$, $p<0.05)$. An additional ANCOVA, adjusted for the influence of age, smoking, alcohol use, and psychopharmacological medication, showed comparable results $(F=3.472, d f=2, p<0.05)$. The BMI was neither included in the ANCOVA nor the following adjusted models because it showed high multicollinearity with the atypical depressive subtype and T, respectively. The adjusted means and SEs are visualized in Figure 2.

Generalized linear regression models were carried out by contrasting the three subgroups, while adjusting for age, smoking, alcohol use, and psychopharmacological medication. The analysis contrasting the atypical depression subgroup with the melancholic subgroup and controls, respectively, showed significant odds ratios (OR) between atypical and melancholic (reference) depression $[\mathrm{OR}=0.024,95 \%$ confidence intervals (CI): 0.001-0.640, $p<0.05$ ] with lower $\mathrm{T}$ levels in the atypical subgroup. Apart from a trend-level association, $\mathrm{T}$ levels did not significantly differ between atypical depression and controls (reference) $(\mathrm{OR}=0.103$, CI: $0.007-1.576, p=0.10)$. No significant difference was found between the $\mathrm{T}$ levels of the melancholic depressives and controls (reference) $(\mathrm{OR}=2.806, \mathrm{CI}: 0.190-41.375, p=0.45)$ (data not tabulated).

\section{Discussion}

This is a preliminary study examining differences of serum $\mathrm{T}$ levels in empirically derived symptom-based depression subtypes and a healthy control group in a Swiss community sample of male adults. The results provided evidence that the atypical depression subtype is discriminable by low serum $\mathrm{T}$ levels in men aged between 21 and 41. First, our results support the notion that the inconsistent evidence from previous studies with respect to 
TABLE 1 | Psychosocial characteristics for men belonging to the depressive subtypes derived from latent class analyses $(n=39)$ and healthy controls ( $n=18)$, unadjusted.

\begin{tabular}{|c|c|c|c|c|}
\hline Characteristic $^{a}$ & $\begin{array}{l}\text { Atypical MDD } \\
n=14\end{array}$ & $\begin{array}{l}\text { Melancholic MDD } \\
\qquad n=25\end{array}$ & $\begin{array}{c}\text { Controls } \\
n=18\end{array}$ & $p$-value \\
\hline & & & & $\begin{array}{l}\text { Chi }{ }^{2} / F \text {-test (two-tailed, overall; post hoc tests } \\
\text { comparing each subtype separately with all others) }\end{array}$ \\
\hline Age & & & & 0.957 \\
\hline 21 & $4(28.6)$ & $3(12.0)$ & $3(16.7)$ & \\
\hline 23 & $2(14.3)$ & $4(16.0)$ & $2(11.1)$ & \\
\hline 28 & $3(21.4)$ & $6(24.0)$ & $5(27.8)$ & \\
\hline 30 & $2(14.3)$ & $4(16.0)$ & $2(11.1)$ & \\
\hline 35 & $2(14.3)$ & $2(8.0)$ & $3(16.7)$ & \\
\hline 41 & $1(7.1)$ & $6(24.0)$ & $3(16.7)$ & \\
\hline Education $^{\mathrm{b}}, n(\%)$ & & & & 0.211 \\
\hline Low & $11(78.6)$ & $16(64.0)$ & $8(47.1)$ & \\
\hline High & $3(21.4)$ & $9(36.0)$ & $9(52.9)$ & \\
\hline Urbanicity $^{\mathrm{c}}, n(\%)$ & & & & 0.222 \\
\hline Urban & $13(92.9)$ & $19(76.0)$ & $12(66.7)$ & \\
\hline Rural & $1(7.1)$ & $6(24.0)$ & $6(33.3)$ & \\
\hline Marital status, $n(\%)$ & & & & 0.608 \\
\hline Unmarried & $9(64.3)$ & $19(76.0)$ & $14(77.8)$ & \\
\hline Married & $4(28.6)$ & $6(24.0)$ & $4(22.2)$ & \\
\hline Divorced & $1(7.1)$ & $0(0.0)$ & $0(0.0)$ & \\
\hline \multicolumn{5}{|l|}{ Comorbidities $^{\mathrm{d}}$ (past year) $n(\%)$} \\
\hline $\mathrm{MDD}^{\mathrm{e}}$ & $6(42.9)$ & $13(52.0)$ & $0(0.0)$ & $<0.001^{r, s}$ \\
\hline Dysthymia ${ }^{\mathrm{e}}$ & $2(14.3)$ & $2(8.0)$ & $0(0.0)$ & 0.267 \\
\hline Hypomania/mania ${ }^{f}$ & $3(21.4)$ & $4(16.0)$ & $0(0.0)$ & 0.105 \\
\hline Bipolar disorder ${ }^{\mathrm{g}}$ & $3(21.4)$ & $2(8.0)$ & $0(0.0)$ & 0.111 \\
\hline Neurasthenia ${ }^{\mathrm{h}, \mathrm{i}}$ & $1(7.1)$ & $6(27.3)$ & $1(5.6)$ & 0.144 \\
\hline GAD & $5(35.7)$ & $2(8.0)$ & $0(0.0)$ & $<0.01^{r}$ \\
\hline Simple phobia ${ }^{f}$ & $5(41.7)$ & $4(19.0)$ & $0(0.0)$ & $<0.05^{r}$ \\
\hline Social phobia ${ }^{f}$ & $3(21.4)$ & $5(23.8)$ & $0(0.0)$ & 0.061 \\
\hline Agoraphobia $^{\dagger}$ & $3(21.4)$ & $0(0.0)$ & $0(0.0)$ & $<0.05^{\mathrm{q}}$ \\
\hline Psychosis syndromes $^{k}$ & $5(35.7)$ & $1(4.0)$ & $0(0.0)$ & $<0.01^{q, r}$ \\
\hline Panic disorder ${ }^{\dagger}$ & $1(8.3)$ & $2(8.3)$ & $0(0.0)$ & 0.443 \\
\hline $\mathrm{OCD}^{f}$ & $1(7.1)$ & $1(4.0)$ & $0(0.0)$ & 0.718 \\
\hline Binge eating ${ }^{\mathrm{f}, \mathrm{I}}$ & $4(28.6)$ & $2(8.0)$ & $1(5.6)$ & 0.144 \\
\hline Anorexia nervosa ${ }^{e}$ & $0(0.0)$ & $1(4.0)$ & $0(0.0)$ & $\mathrm{n} / \mathrm{a}$ \\
\hline Suicide attempt & $1(10.0)$ & $1(5.9)$ & $0(0.0)$ & 0.695 \\
\hline Alcohol dependence $^{f}$ & $2(14.3)$ & $4(16.0)$ & $0(0.0)$ & 0.214 \\
\hline Alcohol abuse $^{f}$ & $1(7.1)$ & $1(4.0)$ & $0(0.0)$ & 0.718 \\
\hline Current smoking & $4(28.6)$ & $11(44.0)$ & $5(27.8)$ & 0.525 \\
\hline Any personality disorder ${ }^{\mathrm{m}}$ & $4(28.6)$ & $1(4.0)$ & $0(0.0)$ & $<0.05^{\mathrm{q}, \mathrm{r}}$ \\
\hline Psychopharmaceuticals, $n^{\text {n }}$ & $4(30.8)$ & $4(23.5)$ & $0(0.0)$ & 0.776 \\
\hline Antidepressant & $4(28.6)$ & $2(8.0)$ & $0(0.0)$ & $<0.05^{r}$ \\
\hline Mood stabilizer & $1(7.1)$ & $1(4.0)$ & $0(0.0)$ & 0.718 \\
\hline Sedative, hypnotic, anxiolytic & $1(7.1)$ & $0(0.0)$ & $0(0.0)$ & 0.246 \\
\hline Neuroleptics & $1(7.1)$ & $0(0.0)$ & $0(0.0)$ & 0.246 \\
\hline Other & $1(7.1)$ & $1(4.0)$ & $0(0.0)$ & 0.718 \\
\hline \multicolumn{4}{|l|}{ Subjective impairment (mean \pm SE) ${ }^{\circ}$} & Kruskal-Wallis test ${ }^{p}$ \\
\hline GSI & $2.25( \pm 0.16)$ & $2.17( \pm 0.07)$ & $1.34( \pm 0.06)$ & $<0.001^{r, s}$ \\
\hline
\end{tabular}

MDD, major depressive disorder; DYST, dysthymia; GAD, generalized anxiety disorder; OCD, obsessive-compulsive disorder; n/a, not available.

${ }^{a}$ The discrepancy between the total number of persons and the number of persons in the following rows result from missing items.

${ }^{b}$ Although the Swiss educational degrees do not entirely correspond with the school system of Anglo-American areas or other countries, the educational degrees were comparatively grouped into the two following categories: low, less than high school diploma; high, high school diploma or higher.

'Urban: Zurich, Winterthur; rural: remaining municipalities of the canton of Zurich.

d12-month prevalence.

e DSM-III-R.

${ }^{f} D S M-I V$.

${ }^{g}$ Def. BRIDGE study (51)

hICD-10.

i3-month criteria.

jDSM-III.

${ }^{k}$ Disorders of form of thought, derealization, depersonalization, delusion, disorder of ego-boundary, hallucinations, paranoia syndrome.

I'ncluding binge eating symptoms.

${ }^{m}$ Paranoid, schizotypal, avoidant, dependent, obsessive-compulsive, borderline, histrionic, narcissistic, and depressive.

${ }^{n}$ Consumption of psychopharmaceuticals during the past 6 months.

${ }^{\circ} \mathrm{Global}$ severity index (GSI) of the SCL-27 (41).

${ }^{p}$ Post hoc tests: pairwise Kruskal-Wallis tests.

${ }^{a}$ Atypical MDD significantly differs from melancholic MDD.

${ }^{r}$ Atypical MDD significantly differs from controls.

${ }^{s}$ Melancholic MDD significantly differs from controls. 


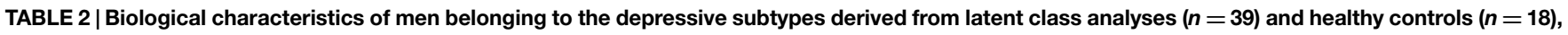
unadjusted.

\begin{tabular}{|c|c|c|c|c|c|}
\hline Biological characteristics & $\begin{array}{c}\text { Atypical MDD } \\
\qquad \begin{array}{c}n=14 \\
\text { Mean (SE) }\end{array}\end{array}$ & $\begin{array}{c}\text { Melancholic MDD } \\
\qquad \begin{array}{c}n=25 \\
\text { Mean (SE) }\end{array}\end{array}$ & $\begin{array}{c}\text { Controls } \\
n=18 \\
\text { Mean (SE) }\end{array}$ & Eta $\left(\eta^{2}\right)$ & $p$-value ${ }^{a}$ \\
\hline Testosterone (ln) & $1.32(0.08)$ & $1.64(0.06)$ & $1.54(0.07)$ & 0.17 & $\leq 0.01^{\mathrm{e},(f)}$ \\
\hline SHBG $(\mathrm{nmol} / \mathrm{L})^{\mathrm{b}}$ & $26.10(4.29)$ & $42.04(2.71)$ & $40.44(4.52)$ & 0.20 & $\leq 0.01^{e,(f)}$ \\
\hline DHEA-S (ug/mL) & $2.17(0.81)$ & $2.48(0.69)$ & $2.31(0.98)$ & 0.02 & 0.513 \\
\hline $\mathrm{LH}(\mathrm{mlU} / \mathrm{ml})^{\mathrm{C}}$ & $2.59(0.56)$ & $3.18(0.37)$ & $2.80(0.61)$ & 0.02 & 0.653 \\
\hline Estradiol (ng/mL) & $28.18(2.10)$ & $26.32(1.57)$ & $26.72(1.85)$ & 0.01 & 0.774 \\
\hline Progesterone (ng/mL) & $0.87(0.14)$ & $1.01(0.11)$ & $0.92(0.13)$ & 0.01 & 0.713 \\
\hline $\mathrm{BMI}^{\mathrm{d}}$ & $29.03(0.92)$ & $23.43(0.67)$ & $23.06(0.78)$ & 0.36 & $\leq 0.001^{e, f}$ \\
\hline Diastolic BP $(\mathrm{mmHg})^{\mathrm{d}}$ & $88.58(2.98)$ & $86.04(2.15)$ & $82.75(2.53)$ & 0.04 & 0.324 \\
\hline Systolic BP $(\mathrm{mmHg})^{\mathrm{d}}$ & $133.23(3.51)$ & $133.10(2.53)$ & $128.94(2.99)$ & 0.02 & 0.515 \\
\hline
\end{tabular}

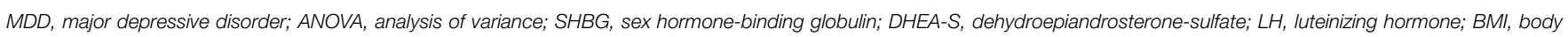
mass index; $B P$, blood pressure.

${ }^{a}$ One-way ANOVANpost hoc tests: Scheffé (homogeneous variances), Games-Howell (non-homogeneous variances).

${ }^{b}$ Missing data: atypical MDD, $n=4$; controls, $n=9$.

${ }^{C}$ Missing data: atypical MDD, $n=3$; controls, $n=9$.

${ }^{d}$ Missing data: atypical MDD, $n=1$.

${ }^{\text {e} A t y p i c a l ~ M D D ~ s i g n i f i c a n t l y ~ d i f f e r s ~ f r o m ~ m e l a n c h o l i c ~ M D D . ~}$

${ }^{f}$ Atypical MDD significantly differs from controls

(f) Parenthesis indicating trend-level associations $p \leq 0.12$.

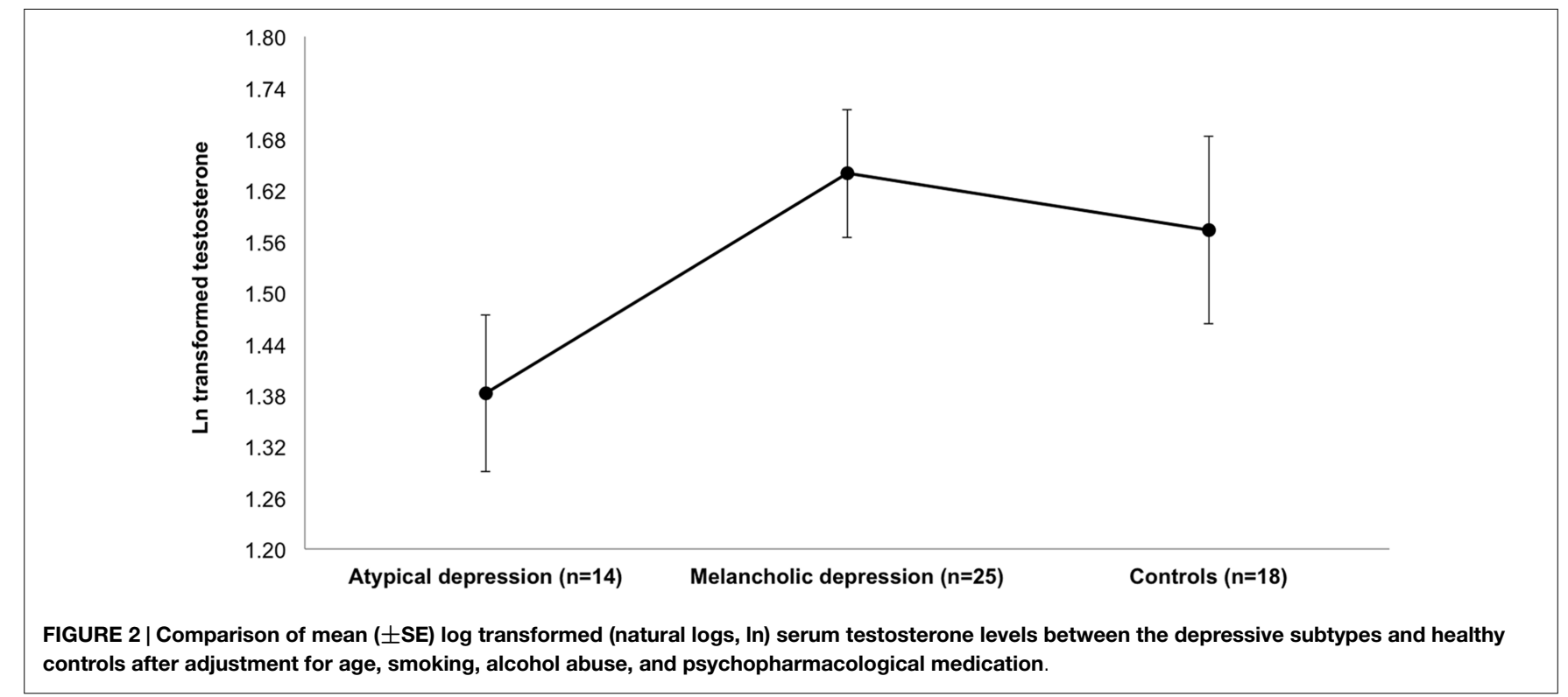

$\mathrm{T}$ levels and MDD may have resulted from the heterogeneity of this diagnosis, while the investigation of more homogeneous symptom-based subtypes provides a more detailed picture. Second, the current analyses confirmed previous findings showing that the melancholic and atypical depressive subtypes were distinguishable by biological correlates $(29,31)$, and expanded this knowledge via the aspect of gonadal hormones.

Our results suggest that the previous findings of low $\mathrm{T}$ levels in depressed men $(4,6,8-10)$ might have resulted from the subgroup of atypical depressives. In fact, the "irritable male syndrome," a behavioral state following withdrawal of $\mathrm{T}$ in adult male mammals (21), shows a certain similarity to the symptoms of atypical depression (30) in human adults. There are several explanations for this specific biological correlate within atypical depressives.
The low $\mathrm{T}$ levels in atypical depressives could be explained by the high BMI associated with this subtype; this marked biological characteristic of atypical depression will be discussed further below. A further conceivable analogy can be drawn from studies demonstrating differences in HPA axis regulation between melancholic and atypical depression $(31,59)$. Melancholic depression was characterized by hypercortisolism while atypical depression was associated with hypocortisolism (31). In combination with the evidence concerning the interactive nature in which the HPA and the HPG axes operate (33) this opens up interesting perspectives. A fairly recent community study on a sample of men showed a positive correlation between endogenous evening saliva $\mathrm{T}$ and overall depression scores, as well as evening cortisol (HPA axis) $(34,60)$. This relation was explained by the effect of cortisol 
modifying the gonadotropin-releasing hormone (GnRH) from the hypothalamus, by influencing the LH from the pituitary or by alterations in the stimulating effect of gonadotropins or gonads $(34,61)$. To return to the depression subtypes, it thus appears plausible that the down-regulated HPA axis is correlated with low $\mathrm{T}$ levels, or even hypogonadism, in atypical depressives. Also, SHBG, which is correlated with T (35), was significantly lower in the atypical depression subtype compared with the melancholic subtype. In line with this hypothesis, the hypercortisolismassociated melancholic subtype should show the opposite, namely $\mathrm{T}$ level alterations.

The initially expected higher T levels in the melancholic depression subtype were based on the available literature concerning the HPA axis dysregulation of this subtype (31) and exogenous $\mathrm{T}$ administration demonstrating that a high $\mathrm{T}$ dose resulted in both depression and hypomania/mania (24-28). In our study, $\mathrm{T}$ levels were actually higher in the melancholic depression subtype compared to both other groups, although they only differed significantly from the atypical group. Therefore, our two depression subtypes were found at both the lowest and highest areas of $\mathrm{T}$ distribution, which would be in line with the parabolic model displaying a curvilinear relationship between $\mathrm{T}$ and depression $(13,15,22)$. However, considering the fact that the T levels of the melancholic subtype were similar to the controls and, moreover, far from significantly differing from the latter (whereas the atypical depression subtype did at least on a trend-level), the clinical relevance of the elevated $\mathrm{T}$ levels in the current study can be questioned. Further studies are needed to replicate the findings of diverging $\mathrm{T}$ levels between melancholic and atypical depression with larger sample sizes.

Beyond the gonadal hormone $\mathrm{T}$ characterizing the atypical depression subtype, the effect size was particularly striking for BMI. In fact, it was more than twice as large as that of $\mathrm{T}$. This is in line with previous findings $(29,36)$ demonstrating a positive association between BMI and atypical depression. A prospective, population-based study revealed that atypical depression was a strong predictor of obesity with an OR of 3.75 (62). Therefore, the BMI seems to be a constitutive characteristic of atypical depression. In the current analysis, however, the simultaneous investigation of $\mathrm{T}$ and BMI was not possible due to inherent model-based constraints combined with the small sample size. Further studies should determine if the low $\mathrm{T}$ levels of the atypical depressive subtype are simply an epiphenomenon of the high BMI associated with this subtype. Moreover, all biological systems/markers (e.g., HPA axis, HPG axis, metabolic abnormalities such as obesity, and inflammatory markers) associated with atypical depression should be balanced in their clinical relevance and better examined with regard to their possible interactions.

Taken together, the atypical depression subtype once again appeared diametrically opposed to the remaining subjects. Apart from its discriminable symptom- and comorbidity profile it also showed specific biological correlates in the current sample of men. It is remarkable that men belonging to the atypical subtype not only exhibited lower $\mathrm{T}$ levels but also, moreover, showed a comorbidity profile usually found in depressive women, that is to say phobias and anxiety. Further studies should show whether our speculation re a feminine profile in atypical depressive men can be confirmed.
Some studies reported a relationship between low $\mathrm{T}$ levels and subthreshold depressive disorders $(15,17,20)$. One epidemiological study reported that lower $\mathrm{T}$ levels are only associated with subthreshold symptoms of anxiety and depression (19). Anxiety symptoms were not included as LCA indicators when deriving the depressive subtypes (37) and the moderate subtype including subjects with a lower severity of depression was excluded from the current analysis in order to increase statistical power. Consequently, we could not further examine this issue. However, an exploratory analysis did not indicate any deviation of the T levels in the moderate depression subgroup.

The following limitations should be noted. First, the sample sizes of the examined subsamples were small and only strong effects achieve common significance levels with small sample sizes. Although we applied robust statistical methods and additionally computed effect sizes, replications of our findings with representative, large samples are necessary. Second, we cannot draw causal conclusions from our data due to the cross-sectional design. The contradictory evidence with regard to causality of the differing $\mathrm{T}$ levels and affective symptoms $(15,63)$ needs further investigation. Hence, the current performance of regression analytic methods implying a causal direction needs to be interpreted as preliminary. Future studies are required to replicate our findings, to longitudinally analyze causal processes [particularly with regard to the interaction between HPG and HPA axes due to their great therapeutic and diagnostic potential (33)], and to assess a broader age range in order to more precisely consider the possibility of age-specific features (64-66). Third, the participants of the laboratory day were selected as a convenience sample. Consequently, a certain selection bias and a restricted external validity cannot be completely excluded. Fourth, we draw analogies between the effects of exogenous high dose $\mathrm{T}$ with endogenous serum $\mathrm{T}$ values although we can not ensure that there is a direct correlation between these biological markers. Fifth, as Jäger et al. (67) emphasized, the comparison of studies examining the issue of $\mathrm{T}$ deficiency is difficult because there is no international consensus on the definition of a standardized normal range of hypogonadism. Sixth, the calculation of free testosterone using total $\mathrm{T}$ and SHGB (68) could be added in future research.

Despite these limitations and its preliminary nature, this community study demonstrated for the first time lower serum $\mathrm{T}$ levels of the empirically derived atypical depression subtype in men. If the current biological findings would be replicated in larger samples, this additional differentiation of depressive subtypes by biological features may indicate distinctive pathophysiological entities and could ameliorate the specificity of depression diagnoses and treatments.

\section{Author Contributions}

SR: designed the study, conducted all statistical analyses, and wrote the manuscript. $\mathrm{MgH}$ : significantly revised the manuscript. $\mathrm{MH}$ : significantly participated in data collection and data management. MM: significantly participated in data collection and data management. AA: significantly participated in data collection and data management of the biological parameters. WR: conceived and designed ZInEP. VA-G: conceived and designed the ZInEP Epidemiology Survey, participated in data management, and 
significantly revised the manuscript. All authors read and accepted the final version of the manuscript.

\section{Acknowledgments}

We thank all the participants in the epidemiological survey as well as the interviewers who collected the data. ZInEP was supported by a private donation. The donator had no further role

\section{References}

1. Rubinow DR, Schmidt PJ. Androgens, brain, and behavior. Am J Psychiatry (1996) 153(8):974-84. doi:10.1176/ajp.153.8.974

2. Kessler RC, Berglund P, Demler O, Jin R, Merikangas KR, Walters EE. Lifetime prevalence and age-of-onset distributions of DSM-IV disorders in the National Comorbidity Survey replication. Arch Gen Psychiatry (2005) 62(6):593-602. doi:10.1001/archpsyc.62.6.617

3. Ebinger M, Sievers C, Ivan D, Schneider HJ, Stalla GK. Is there a neuroendocrinological rationale for testosterone as a therapeutic option in depression? J Psychopharmacol (2009) 23(7):841-53. doi:10.1177/0269881108092337

4. Shores MM, Sloan KL, Matsumoto AM, Moceri VM, Felker B, Kivlahan DR. Increased incidence of diagnosed depressive illness in hypogonadal older men. Arch Gen Psychiatry (2004) 61(2):162-7. doi:10.1001/archpsyc.61.2.162

5. Morsink LF, Vogelzangs N, Nicklas BJ, Beekman AT, Satterfield S, Rubin SM, et al. Associations between sex steroid hormone levels and depressive symptoms in elderly men and women: results from the health ABC study. Psychoneuroendocrinology (2007) 32(8-10):874-83. doi:10.1016/j.psyneuen.2007.06.009

6. Joshi D, van Schoor NM, de Ronde W, Schaap LA, Comijs HC, Beekman AT, et al. Low free testosterone levels are associated with prevalence and incidence of depressive symptoms in older men. Clin Endocrinol (Oxf) (2010) 72(2):232-40. doi:10.1111/j.1365-2265.2009.03641.x

7. Shores MM, Moceri VM, Sloan KL, Matsumoto AM, Kivlahan DR. Low testosterone levels predict incident depressive illness in older men: effects of age and medical morbidity. J Clin Psychiatry (2005) 66(1):7-14. doi:10.4088/JCP. v66n0102

8. Schweiger U, Deuschle M, Weber B, Korner A, Lammers CH, Schmider $\mathrm{J}$, et al. Testosterone, gonadotropin, and cortisol secretion in male patients with major depression. Psychosom Med (1999) 61(3):292-6. doi:10.1097/ 00006842-199905000-00007

9. Seidman SN, Walsh BT. Testosterone and depression in aging men. Am J Geriatr Psychiatry (1999) 7(1):18-33. doi:10.1097/00019442-199902000-00004

10. Zitzmann M. Testosterone and the brain. Aging Male (2006) 9(4):195-9. doi:10. 1080/13685530601040679

11. Kaneda Y, Fujii A. No relationship between testosterone levels and depressive symptoms in aging men. Eur Psychiatry (2002) 17(7):411-3. doi:10.1016/ S0924-9338(02)00694-6

12. Levitt AJ, Joffe RT. Total and free testosterone in depressed men. Acta Psychiatr Scand (1988) 77(3):346-8. doi:10.1111/j.1600-0447.1988.tb05132.x

13. Seidman SN. Normative hypogonadism and depression: does 'andropause' exist? Int J Impot Res (2006) 18(5):415-22. doi:10.1038/sj.ijir.3901443

14. Granger DA, Shirtcliff EA, Booth A, Kivlighan KT, Schwartz EB. The "trouble" with salivary testosterone. Psychoneuroendocrinology (2004) 29(10):1229-40. doi:10.1016/j.psyneuen.2004.02.005

15. Johnson JM, Nachtigall LB, Stern TA. The effect of testosterone levels on mood in men: a review. Psychosomatics (2013) 54(6):509-14. doi:10.1016/j.psym. 2013.06.018

16. Amiaz R, Seidman SN. Testosterone and depression in men. Curr Opin Endocrinol Diabetes Obes (2008) 15(3):278-83. doi:10.1097/MED. $0 \mathrm{~b} 013 \mathrm{e} 3282 \mathrm{fc} 27 \mathrm{eb}$

17. Seidman SN, Araujo AB, Roose SP, Devanand DP, Xie S, Cooper TB, et al. Low testosterone levels in elderly men with dysthymic disorder. Am J Psychiatry (2002) 159(3):456-9. doi:10.1176/appi.ajp.159.3.456

18. Seidman SN. Testosterone deficiency and mood in aging men: pathogenic and therapeutic interactions. World J Biol Psychiatry (2003) 4(1):14-20. doi:10.3109/ 15622970309167905

19. Berglund LH, Prytz HS, Perski A, Svartberg J. Testosterone levels and psychological health status in men from a general population: the Tromso study. Aging Male (2011) 14(1):37-41. doi:10.3109/13685538.2010.522276 in experimental design; the collection, analysis, and interpretation of data; the writing of this report; or the decision to submit this paper for publication. The ZInEP Epidemiology Survey, in particular research in the sociophysiological lab, was supported by the Swiss National Science Foundation (grant \# 3247B0-122071). The population data were provided by the Swiss Federal Office of Statistics. The second author was supported by a grant from the Swiss National Science foundation (SNSF; PP00P1-123377/1).

20. Shores MM, Kivlahan DR, Sadak TI, Li EJ, Matsumoto AM. A randomized, double-blind, placebo-controlled study of testosterone treatment in hypogonadal older men with subthreshold depression (dysthymia or minor depression). J Clin Psychiatry (2009) 70(7):1009-16. doi:10.4088/JCP.08m04478

21. Lincoln GA. The irritable male syndrome. Reprod Fertil Dev (2001) 13(7-8):567-76. doi:10.1071/RD01077

22. Booth A, Johnson DR, Granger DA. Testosterone and men's depression: the role of social behavior. J Health Soc Behav (1999) 40(2):130-40. doi:10.2307/ 2676369

23. Talih F, Fattal O, Malone D Jr. Anabolic steroid abuse: psychiatric and physical costs. Cleve Clin J Med (2007) 74(5):341-4, 346, 349-52. doi:10.3949/ccjm.74. 5.341

24. Pope HG Jr, Katz DL. Psychiatric and medical effects of anabolic-androgenic steroid use. A controlled study of 160 athletes. Arch Gen Psychiatry (1994) 51(5):375-82. doi:10.1001/archpsyc.1994.03950050035004

25. Malone DA Jr, Dimeff RJ, Lombardo JA, Sample RH. Psychiatric effects and psychoactive substance use in anabolic-androgenic steroid users. Clin J Sport Med (1995) 5(1):25-31. doi:10.1097/00042752-199501000-00005

26. Pope HG Jr, Kouri EM, Hudson JI. Effects of supraphysiologic doses of testosterone on mood and aggression in normal men: a randomized controlled trial. Arch Gen Psychiatry (2000) 57(2):133-40. doi:10.1001/archpsyc.57.2.133

27. Kouri EM, Lukas SE, Pope HG Jr, Oliva PS. Increased aggressive responding in male volunteers following the administration of gradually increasing doses of testosterone cypionate. Drug Alcohol Depend (1995) 40(1):73-9. doi:10.1016/ 0376-8716(95)01192-7

28. Su TP, Pagliaro M, Schmidt PJ, Pickar D, Wolkowitz O, Rubinow DR. Neuropsychiatric effects of anabolic steroids in male normal volunteers. JAMA (1993) 269(21):2760-4. doi:10.1001/jama.269.21.2760

29. Lamers F, Vogelzangs N, Merikangas KR, de Jonge P, Beekman AT, Penninx BW. Evidence for a differential role of HPA-axis function, inflammation and metabolic syndrome in melancholic versus atypical depression. Mol Psychiatry (2013) 18(6):692-9. doi:10.1038/mp.2012.144

30. Halbreich U, Kahn LS. Atypical depression, somatic depression and anxious depression in women: are they gender-preferred phenotypes? J Affect Disord (2007) 102(1-3):245-58. doi:10.1016/j.jad.2006.09.023

31. Baumeister H, Parker G. Meta-review of depressive subtyping models. J Affect Disord (2012) 139:126-40. doi:10.1016/j.jad.2011.07.015

32. Young E, Korszun A. Sex, trauma, stress hormones and depression. Mol Psychiatry (2010) 15(1):23-8. doi:10.1038/mp.2009.94

33. Viau V. Functional cross-talk between the hypothalamic-pituitary-gonadal and -adrenal axes. J Neuroendocrinol (2002) 14(6):506-13. doi:10.1046/j.1365-2826. 2002.00798.x

34. Sigurdsson B, Palsson SP, Aevarsson O, Olafsdottir M, Johannsson M. Saliva testosterone and cortisol in male depressive syndrome, a community study. The Sudurnesjamenn study. Nord J Psychiatry (2014) 68(8):579-87. doi:10.3109/ 08039488.2014.898791

35. Zitzmann M, Nieschlag E. Testosterone levels in healthy men and the relation to behavioural and physical characteristics: facts and constructs. Eur J Endocrinol (2001) 144(3):183-97. doi:10.1530/eje.0.1440183

36. Kendler KS, Eaves LJ, Walters EE, Neale MC, Heath AC, Kessler RC. The identification and validation of distinct depressive syndromes in a populationbased sample of female twins. Arch Gen Psychiatry (1996) 53(5):391-9. doi:10. 1001/archpsyc.1996.01830050025004

37. Rodgers S, Grosse Holtforth M, Muller M, Hengartner MP, Rossler W, AjdacicGross V. Symptom-based subtypes of depression and their psychosocial correlates: a person-centered approach focusing on the influence of sex. $J$ Affect Disord (2014) 156:92-103. doi:10.1016/j.jad.2013.11.021

38. Angst J, Gamma A, Neuenschwander M, Ajdacic-Gross V, Eich D, Rossler W, et al. Prevalence of mental disorders in the Zurich cohort study: a twenty 
year prospective study. Epidemiol Psichiatr Soc (2005) 14(2):68-76. doi:10.1017/ S1121189X00006278

39. Ajdacic-Gross V, Muller M, Rodgers S, Warnke I, Hengartner MP, Landolt K, et al. The ZInEP Epidemiology Survey: background, design and methods. Int $J$ Methods Psychiatr Res (2014) 23(4):451-68. doi:10.1002/mpr.1441

40. Rössler W, Riecher-Rössler A, Angst J, Murray R, Gamma A, Eich D, et al. Psychotic experiences in the general population: a twenty-year prospective community study. Schizophr Res (2007) 92(1-3):1-14. doi:10.1016/j.schres. 2007.01.002

41. Hardt J, Egle UT, Kappis B, Hessel A, Brahler E. [Symptom checklist SCL27]. Psychother Psychosom Med Psychol (2004) 54(5):214-23. doi:10.1055/ s-2003-814786

42. Angst J, Dobler-Mikola A, Binder J. The Zurich study - a prospective epidemiological study of depressive, neurotic and psychosomatic syndromes. I. Problem, methodology. Eur Arch Psychiatry Neurol Sci (1984) 234(1):13-20. doi:10.1007/BF00432878

43. Lamers F, de Jonge P, Nolen WA, Smit JH, Zitman FG, Beekman AT, et al. Identifying depressive subtypes in a large cohort study: results from the Netherlands study of depression and anxiety (NESDA). J Clin Psychiatry (2010) 71(12):1582-9. doi:10.4088/JCP.09m05398blu

44. Lamers F, Rhebergen D, Merikangas KR, de Jonge P, Beekman AT, Penninx BW. Stability and transitions of depressive subtypes over a 2-year follow-up. Psychol Med (2012) 42(10):2083-93. doi:10.1017/S0033291712000141

45. Delhez M, Hansenne M, Legros JJ. Andropause and psychopathology: minor symptoms rather than pathological ones. Psychoneuroendocrinology (2003) 28(7):863-74. doi:10.1016/S0306-4530(02)00102-6

46. Sullivan PF, Prescott CA, Kendler KS. The subtypes of major depression in a twin registry. J Affect Disord (2002) 68(2-3):273-84. doi:10.1016/ S0165-0327(00)00364-5

47. APA. American Psychiatric Association: Diagnostic and Statistical Manual of Mental Disorders - DSM-V. 5th ed. Arlington, VA: American Psychiatric Association (2013).

48. APA. American Psychiatric Association: Diagnostic and Statistical Manual of Mental Disorders - DSM-IV-TR. 4th ed. Washington, DC: American Psychiatric Association (2000).

49. APA. American Psychiatric Association: Diagnostic and Statistical Manual of Mental Disorders. 3rd ed. Washington, DC: American Psychiatric Association (1987).

50. WHO. The ICD-10 Classification of Mental and Behavioural Disorders: Clinical Descriptions and Diagnostic Guidelines. Geneva: World Health Organization (1992).

51. Angst J, Azorin JM, Bowden CL, Perugi G, Vieta E, Gamma A, et al. Prevalence and characteristics of undiagnosed bipolar disorders in patients with a major depressive episode: the BRIDGE study. Arch Gen Psychiatry (2011) 68(8):791-8. doi:10.1001/archgenpsychiatry.2011.87

52. Doering S, Renn D, Höfer S, Rumpold G, Smrekar U, Janecke N, et al. [Validation of the "assessment of DSM-IV personality disorders (ADP-IV)" questionnaire]. Z Psychosom Med Psychother (2007) 53(2):111-28.

53. Schotte CKW, de Doncker D. ADP-IV Questionnaire. Antwerp: University Hospital Antwerp (1994).

54. Schotte CK, De Doncker DA, Dmitruk D, Van Mulders I, D’Haenen H, Cosyns P. The ADP-IV questionnaire: differential validity and concordance with the semi-structured interview. J Pers Disord (2004) 18(4):405-19. doi:10.1521/pedi. 2004.18.4.405

55. Brambilla DJ, Matsumoto AM, Araujo AB, McKinlay JB. The effect of diurnal variation on clinical measurement of serum testosterone and other sex hormone levels in men. J Clin Endocrinol Metab (2009) 94(3):907-13. doi:10.1210/jc. 2008-1902

56. Cohen J. Statistical Power Analysis for the Behavioral Sciences. 2 ed. New York, NY: Lawrence Erlbaum Associates (1988).

57. Heritier S, Cantoni E, Copt S, Victoria-Feser M-P. Robust Methods in Biostatistics. Chippenham: Wiley (2009).

58. Arver S, Lehtihet M. Current guidelines for the diagnosis of testosterone deficiency. Front Horm Res (2009) 37:5-20. doi:10.1159/000175839

59. Gold PW, Chrousos GP. Organization of the stress system and its dysregulation in melancholic and atypical depression: high vs low CRH/NE states. $\mathrm{Mol}$ Psychiatry (2002) 7(3):254-75. doi:10.1038/sj.mp.4001032

60. Sigurdsson B, Palsson SP, Aevarsson O, Olafsdottir M, Johannsson M. Saliva testosterone and male depressive syndrome in a commonity study: the Sudurnesjamenn study. Eur Psychiatry (2013) 28(Suppl 1):1. doi:10.3109/08039488. 2014.898791

61. Rivier C, Rivest S. Effect of stress on the activity of the hypothalamicpituitary-gonadal axis: peripheral and central mechanisms. Biol Reprod (1991) 45(4):523-32. doi:10.1095/biolreprod45.4.523

62. Lasserre AM, Glaus J, Vandeleur CL, Marques-Vidal P, Vaucher J, Bastardot F, et al. Depression with atypical features and increase in obesity, body mass index, waist circumference, and fat mass: a prospective, population-based study. JAMA Psychiatry (2014) 71(8):880-8. doi:10.1001/jamapsychiatry.2014.411

63. Giltay EJ, Enter D, Zitman FG, Penninx BW, van Pelt J, Spinhoven P, et al. Salivary testosterone: associations with depression, anxiety disorders, and antidepressant use in a large cohort study. J Psychosom Res (2012) 72(3):205-13. doi:10.1016/j.jpsychores.2011.11.014

64. Sher L. High and low testosterone levels may be associated with suicidal behavior in young and older men, respectively. Aust N Z J Psychiatry (2013) 47(5):492-3. doi:10.1177/0004867412463976

65. Sher L. Low testosterone levels may be associated with suicidal behavior in older men while high testosterone levels may be related to suicidal behavior in adolescents and young adults: a hypothesis. Int J Adolesc Med Health (2013) 25(3):263-8. doi:10.1515/ijamh-2013-0060

66. Diver MJ, Imtiaz KE, Ahmad AM, Vora JP, Fraser WD. Diurnal rhythms of serum total, free and bioavailable testosterone and of SHBG in middle-aged men compared with those in young men. Clin Endocrinol (Oxf) (2003) 58(6):710-7. doi:10.1046/j.1365-2265.2003.01772.x

67. Jager T, Kramer J, Batz O, Rubben H, von Ostau C, Szarvas T. [Testosterone deficiency - an underestimated risk for men? Prevalence of hypogonadism]. Urologe A (2013) 52(12):1684-9. doi:10.1007/s00120-013-3203-8

68. Ly LP, Handelsman DJ. Empirical estimation of free testosterone from testosterone and sex hormone-binding globulin immunoassays. Eur J Endocrinol (2005) 152(3):471-8. doi:10.1530/eje.1.01844

Conflict of Interest Statement: The authors declare that the research was conducted in the absence of any commercial or financial relationships that could be construed as a potential conflict of interest.

Copyright (c) 2015 Rodgers, grosse Holtforth, Hengartner, Müller, Aleksandrowicz, Rössler and Ajdacic-Gross. This is an open-access article distributed under the terms of the Creative Commons Attribution License (CC BY). The use, distribution or reproduction in other forums is permitted, provided the original author(s) or licensor are credited and that the original publication in this journal is cited, in accordance with accepted academic practice. No use, distribution or reproduction is permitted which does not comply with these terms. 